\title{
ON FAITHFUL REPRESENTATIONS OF LIE GROUPS
}

\section{HARISH-CHANDRA}

Let $G$ and $H$ be two connected Lie groups and $\phi$ a continuous homomorphism of $H$ into the group of automorphisms of $G$. Then we define a new group $G \times{ }_{\phi} H$ as follows. The elements of $G \times_{\phi} H$ are pairs $(g, h)(g \in G, h \in H)$ and group multiplication is defined by

$$
\left(g_{1}, h_{1}\right)\left(g_{2}, h_{2}\right)=\left(g_{1}\left(\phi\left(h_{1}\right) g_{2}\right), h_{1} h_{2}\right) \text {. }
$$

Topologically $G \times{ }_{\phi} H$ is taken to be just the Cartesian product of $G$ and $H$. It is then easily proved that under this topology $G \times_{\phi} H$ is a Lie group. It is called the semidirect product of $G$ and $H$ under $\phi$. The object of this note is to prove the following theorem.

THEOREM. Let $G$ be a connected, simply connected solvable Lie group and $H$ a connected Lie group which has a faithful representation. Let $\phi$ be any continuous homomorphism of $H$ into the group of automorphisms of $G$. Then $G \times_{\phi} H$ has a faithful representation.

The special case of this theorem when $H$ is semisimple is due to Cartan. ${ }^{1}$

Let $R$ be the field of real numbers and $K$ the field of either real or complex numbers. Let $G$ be a connected Lie group with the Lie algebra $g$ and $\theta$ a representation of $G$ over $K$ of degree $\dot{d}$. Then we denote by $d \theta$ the representation of $\mathfrak{g}$ given by $^{2}$

$$
d \theta(x)=\lim _{t \rightarrow 0} \frac{\theta(\exp t X)-I}{t}
$$

where $t \in R$ and $I$ is the unit matrix of degree $\dot{d}$. Let $G L(K, \dot{d})$ denote the group of all nonsingular matrices of degree $\dot{d}$ with coefficients in $K$. Any subgroup of $G L(K, \dot{d})$ will be called a linear group of degree $\dot{d}$. Let $\theta$ be the identity representation of a linear Lie group $G$ with the Lie algebra $g$ so that $\theta(x)=x(x \in G)$. Then $d \theta$ is a faithful representation of $g$ and $\exp d \theta(X)=\theta(\exp X)=\exp X$ for any $x \in g$. Hence we may identify $\mathfrak{g}$ with $d \theta(\mathfrak{g})$ under $d \theta$. $g$ can therefore be regarded as a linear Lie algebra. In particular the Lie algebra of $G L(K, \dot{d})$ then

Received by the editors October 4, 1948, and, in revised form, February 11, 1949.

1 Cartan, J. Math. Pures Appl. vol. 17 (1938) pp. 1-12. See also Malcev, C. R. (Doklady) Acad. Sci. URSS. vol. 40 (1943) pp. 87-89.

2 For the precise definitions of the terms used in this paper see Chevalley, Theory of Lie groups, Princeton University Press, 1946. 
consists of all matrices of degree $\dot{d}$ with coefficients in $K$. We denote it by $\mathfrak{g l}(K, \dot{d})$. Given any subalgebra $\mathfrak{h} C \mathfrak{g l}(K, \dot{d})$, by the linear Lie group generated by $\mathfrak{h}$ we mean the analytic subgroup of $G L(K, \dot{d})$ corresponding to $\mathfrak{h}$.

Let us call a matrix subtriangular if it has zeros on and below the the diagonal. First we state the following two well known lemmas.

LEMMA $1 .^{8}$ Let $\mathfrak{\Re}$ be the Lie algebra of all subtriangular $\dot{d} \times \dot{d}$ matrices

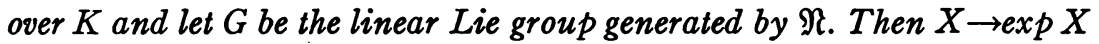
is a topological mapping of $\mathfrak{N}$ onto $G$. Also $G$ consists of all matrices which have zeros below the diagonal and 1 everywhere on the diagonal.

LEMMA 2.4 Let $G$ be a connected, simply connected solvable Lie group. Then every analytic subgroup of $G$ is closed and simply connected.

From now on I adhere strictly to the notation of my paper, Faithful representations of Lie algebras, ${ }^{b}$ which will be quoted as FRL.

LEMMA 3. Let $\mathfrak{R}, \mathfrak{R}, \mathfrak{D}$ be as in Lemma 1 of FRL. We construct the faithful representation $\theta$ of $\mathfrak{Q}+\mathfrak{D}$ as described there. Then for any $Y_{1}, \cdots, Y_{\imath} \in \mathbb{R}$ and $D_{1}, \cdots, D_{\imath} \in \mathfrak{D}$,

(1) $\exp \theta\left(Y_{1}\right) \cdots \exp \theta\left(Y_{\imath}\right) \neq I$ unless $\exp Y_{1} \cdots \exp Y_{\imath}=I^{\prime \prime}$,

(2) $\exp \theta\left(D_{1}\right) \cdots \exp \theta\left(D_{s}\right)=I$

if and only if $\exp D_{1} \cdots \exp D_{\varepsilon}=I^{\prime}$,

where $I, I^{\prime}$, and $I^{\prime \prime}$ are unit matrices of suitable degrees.

If we use the notation of the proof of Lemma 1 of FRL, (1) follows immediately from the fact that $\mathfrak{A} / \mathfrak{X} \cong \mathfrak{Y}^{*} / \mathfrak{X}^{*}$ where $\mathfrak{X}^{*}=\mathfrak{X} / \mathfrak{X}_{0}$. Now we prove (2). Put $\omega^{*}(X)=(\omega(X))^{*}$ for $X \in \mathbb{R}$. Then it is easily proved by induction on $s$ that for any $D \in \mathscr{D}$

$$
\{\theta(D)\}^{*} \omega^{*}(X)=\omega^{*}\left(D^{*} X\right), \quad s \geqq 1 .
$$

Hence $(\exp \theta(D)) \omega^{*}(X)=\omega^{*}((\exp D) X)$. Also since $\theta(D)=d_{D}^{*}$ is a derivation of $\mathfrak{A}^{*}=\mathfrak{A} / \mathfrak{X}_{0}, \exp \theta(D)$ is an automorphism of $\mathfrak{A}^{*}$. Put $Y_{\mathbf{X}}=\left(\exp D_{1}\right) \cdots\left(\exp D_{s}\right) X(X \in \mathbb{R})$. Then if

$$
\exp \theta\left(D_{1}\right) \cdots \exp \theta\left(D_{s}\right)=I,
$$

$\omega^{*}\left(Y_{X}\right)-\omega^{*}(X)=0$ for every $X \in \mathbb{R}$. Hence $\omega\left(Y_{X}-X\right) \in \mathfrak{X}_{0} \subset \mathfrak{X}$. Therefore $\pi \omega\left(Y_{X}-X\right)=Y_{X}-X=0$. Since this is true for every $X$,

${ }^{3}$ Birkhoff, Ann. of Math. vol. 38 (1937) pp. 526-532.

${ }^{4}$ Chevalley, Ann. of Math. vol. 42 (1941) pp. 668-675.

${ }^{5}$ Harish-Chandra, Ann. of Math. vol. 50 (1949) pp. 68-76. 
$\left(\exp D_{1}\right) \cdots\left(\exp D_{\mathfrak{s}}\right)=I^{\prime}$. The converse is obvious. Hence the lemma is proved.

Lemma 4. Let $G$ be a connected, simply connected solvable Lie group with Lie algebra $\mathfrak{g}$. Let $\mathfrak{\Re}$ be the maximal nilpotent ideal of $\mathfrak{g}$. Then $G$ has a faithful representation $\psi$ such that $d \Psi(X)$ is nilpotent for every $X \in \mathfrak{R}$.

By Corollary 1 of FRL we can find a faithful representation $\rho_{0}$ of $g$ such that $\rho_{0}(X)$ is nilpotent for every $X \in \mathfrak{R}$. We can therefore choose, if necessary, a new base in our representation space such that with respect to this base the matrix representing $\rho_{0}(X)$ is subtriangular for every $X \in \mathfrak{N}$. Now consider the factor algebra $\mathfrak{g} / \mathfrak{N}$ which is abelian and hence nilpotent. By the same corollary it follows that $\mathfrak{g} / \mathfrak{N}$ has a faithful representation by nilpotent matrices. Hence $g$ has a representation $\rho_{1}$ such that the kernel of $\rho_{1}$ is $\mathfrak{N}$ and $\rho_{1}(X)$ is nilpotent for all $X \in g$. We can again arrange that $\rho_{1}(X)$ is subtriangular for all $X$. Put $\rho=\rho_{0} \dot{+} \rho_{1}$, where $\dot{+}$ denotes direct sum. Since $G$ is simply connected, there exist representations $\psi_{0}$ and $\psi_{1}$ of $G$ such that $d \psi_{0}=\rho_{0}, d \psi_{1}=\rho_{1}$. Put $\psi=\psi_{0}+\psi_{1}$. Then $d \psi=\rho$. Let $N$ be the analytic subgroup of $G$ corresponding to $\mathfrak{R}$. Then from Lemma 2, $N$ is a closed invariant subgroup. Consider $\psi(N)$. It is clear that $d \psi(Y)=\rho_{0}(Y)+\rho_{1}(Y)$ is subtriangular for all $Y \in \mathfrak{R}$. Hence from Lemmas 1 and 2 it follows that the linear Lie group $\psi(N)$ generated by $d \psi(\mathfrak{R})$ is simply connected. Since $d \psi$ is an isomorphism, $\psi(N)$ is locally isomorphic to $N$. Therefore since $\psi(N)$ is simply connected, $\psi$ maps $N$ isomorphically. Similarly we prove that $\psi_{1}(G)$ is simply connected. It is clear that the kernel of $\psi_{1}$ contains $N$. Hence $\psi_{1}$ defines a representation $\psi^{*}$ of $G / N$ given by $\psi^{*}\left(x^{*}\right)=\psi_{1}(x)$ where $x \rightarrow x^{*}$ is the natural homomorphism of $G$ onto $G / N=G^{*}$. Since $d \psi^{*}$ is an isomorphism, the kernel of $d \psi_{1}=\rho_{1}$ being $\mathfrak{N}$, it follows from the simple connectivity of $\psi^{*}\left(G^{*}\right)=\psi_{1}(G)$ that $\psi^{*}$ is an isomorphism. Hence the kernel of $\psi_{1}$ is exactly $N$. Let $D$ be the kernel of $\psi$. Then $D$ is contained in the kernel of $\psi_{1}$ which is $N$. Also since $\psi$ is faithful on $N, D \cap N=\{e\}$ where $e$ is the unit element of $N$. Hence $D=\{e\}$ and $\psi$ is a faithful representation. Also $d \psi(X)$ is nilpotent for every $X \in \mathfrak{R}$.

Now we come to the proof of the theorem. Let $g$ be the Lie algebra of $G$ and $\mathfrak{R}$ the maximal nilpotent ideal of $g$. By Lemma $4, G$ has a faithful representation. Hence we may assume that $G$ is a linear Lie group such that every element of $\mathfrak{N}$ is nilpotent. We keep to the notation of Lemma 3 except that $\mathfrak{R}$ is replaced by $\mathfrak{g}$. Let $\mathfrak{h}$ be the Lie algebra of $H$. Define a homomorphism $d \tau$ of $\mathfrak{h}$ into $\mathfrak{D}$ as follows. Let 
Aut $(G)$ be the group of automorphisms of $G$. Then Aut $(G)$ is a Lie group with a Lie algebra $\mathfrak{A}$. It is well known that there exists an isomorphism $\lambda$ of $\mathfrak{A}$ onto $\mathfrak{D}$ such that

$$
(\exp A) \exp X=\exp ((\exp \lambda(A)) X)
$$

for any $A \in \mathcal{A}$ and $X \in \mathfrak{g}$. We put $d \tau=\lambda \circ d \phi$ where $d \phi$ is the homomorphism of $\mathfrak{h}$ into $\mathfrak{A}$ induced by $\phi$. Then for any $P_{\mathfrak{i}} \in \mathfrak{h}, 1 \leqq i \leqq r$,

$$
\phi\left(\exp P_{1} \cdots \exp P_{r}\right) \exp X=\exp \left(\left(\exp d \tau\left(P_{1}\right) \cdots \exp d \tau\left(P_{r}\right)\right) X\right) \text {. }
$$

Let $e$ and $e^{\prime}$ denote the identity elements of $G$ and $H$ respectively. Suppose $\exp P_{1} \cdots \exp P_{r}=e^{\prime}$. Then clearly

$$
\begin{aligned}
\exp X & =\phi\left(\exp P_{1} \cdots \exp P_{r}\right) \exp X \\
& =\exp \left(\left(\exp d \tau\left(P_{1}\right) \cdots \exp d \tau\left(P_{r}\right)\right) X\right) .
\end{aligned}
$$

Since this is true for every $X \in \mathfrak{g}$,

$$
\exp d \tau\left(P_{1}\right) \cdots \exp d \tau\left(P_{r}\right)=I^{\prime} .
$$

Hence we can define a representation $\tau$ of $H$ by the rule $\tau\left(\exp P_{1} \cdots \exp P_{r}\right)=\exp d \tau\left(P_{1}\right) \cdots \exp d \tau\left(P_{r}\right),\left(P_{1}, \cdots, P_{r} \in \mathfrak{h}\right)$.

Put $d \psi=\theta$ odr. Then $d \psi$ is a representation of $\mathfrak{h}$. Suppose $\exp P_{1} \ldots$ $\exp P_{r}=e^{\prime}\left(P_{1}, \cdots, P_{r} \in \mathfrak{h}\right)$. Then

$$
\tau\left(\exp P_{1} \cdots \exp P_{r}\right)=\exp d \tau\left(P_{1}\right) \cdots \exp d \tau\left(P_{r}\right)=I^{\prime}
$$

and from Lemma 3

$$
\exp d \psi\left(P_{1}\right) \cdots \exp d \psi\left(P_{r}\right)=I .
$$

Hence we can again define a representation $\psi$ of $H$ by putting $\psi\left(\exp P_{1} \cdots \exp P_{r}\right)=\exp d \psi\left(P_{1}\right) \cdots \exp d \psi\left(P_{r}\right) \quad\left(P_{1}, \cdots, P_{r} \in \mathfrak{h}\right)$. Also since $G$ is simply connected there exists a representation $\chi$ of $G$ such that $d \chi(X)=\theta(X)$ for every $X \in \mathfrak{g}$. Hence

$$
\chi\left(\exp Y_{1} \cdots \exp Y_{r}\right)=\exp \theta\left(Y_{1}\right) \cdots \exp \theta\left(Y_{r}\right) \quad\left(Y_{i} \in \mathbb{S}, 1 \leqq i \leqq r\right) .
$$

From Lemma 3 it follows that $\chi$ is faithful.

Consider the mapping $\mu$ of $G \times_{\phi} H$ defined by $\mu(g, h)=\chi(g) \psi(h)$. We claim that $\mu$ is a representation. For any $P \in \mathfrak{h}$ and $X \in \mathfrak{g}$ consider $\psi(\exp P) \chi(\exp X)(\psi(\exp P))^{-1}=\exp d \psi(P) \exp \theta(X) \exp (-d \psi(P))$

$$
=\exp \theta(D) \exp \theta(X) \exp (-\theta(D))
$$

where $D=d \tau(P)$. Now for any two elements $A, B \in \mathfrak{g l}(K, d)$, 


$$
\exp A \exp B \exp (-A)=\exp ((\exp \operatorname{ad} A) B)
$$

where ad $A$ is defined as in FRL. Since $[\theta(D), \theta(Y)]=\theta([D, Y])$ $=\theta(D Y)$ for any $Y \in \mathfrak{g}$, it follows immediately that

$$
\begin{aligned}
\exp \theta(D) \exp \theta(X) \exp (-\theta(D)) & =\exp \theta((\exp D) X) \\
& =\exp \theta(\tau(\exp P) X) \\
& =\chi(\exp \tau(\exp P) X) \\
& =\chi(\phi(\exp P) \exp X) .
\end{aligned}
$$

Since any $h \in H$ can be written in the form $\exp P_{1} \cdots \exp P_{r}$, $P_{i} \in \mathfrak{h}, 1 \leqq i \leqq r, r \geqq 1$, we get

$$
\psi(h) \chi(\exp X)(\psi(h))^{-1}=\chi(\phi(h) \exp X) .
$$

Similarly since every $g \in G$ can be written as $\exp Y_{1} \cdots \exp Y_{r}$, $Y_{i} \in \mathfrak{g}, 1 \leqq i \leqq r, r \geqq 1$, we have

$$
\psi(h) \chi(g)(\psi(h))^{-1}=\chi(\phi(h) g) .
$$

Therefore

$$
\begin{aligned}
\mu\left(\left(g_{1}, h_{1}\right)\left(g_{2}, h_{2}\right)\right) & =\mu\left(g_{1} \phi\left(h_{1}\right) g_{2}, h_{1} h_{2}\right)=\chi\left(g_{1} \phi\left(h_{1}\right) g_{2}\right) \psi\left(h_{1} h_{2}\right) \\
& =\chi\left(g_{1}\right) \chi\left(\phi\left(h_{1}\right) g_{2}\right) \psi\left(h_{1}\right) \psi\left(h_{2}\right) \\
& =\chi\left(g_{1}\right) \psi\left(h_{1}\right) \chi\left(g_{2}\right) \psi\left(h_{2}\right)=\mu\left(g_{1}, h_{1}\right) \mu\left(g_{2}, h_{2}\right) .
\end{aligned}
$$

Since $\mu$ is clearly a continuous mapping it is a representation of $G \times_{\phi} H$. By hypothesis $H$ has a faithful representation $\nu_{0}$. Define a representation $\nu$ of $G \times_{\phi} H$ by $\nu(g, h)=\nu_{0}(h)$ and put $\xi=\mu+\nu$. Suppose $(g, h)$ belongs to the kernel of $\xi$. Then since $\xi(g, h)=\mu(g, h) \dot{+} \nu_{0}(h)$ $=\chi(g) \psi(h)+\nu_{0}(h)$ and since $\nu_{0}$ is faithful on $H, h=e^{\prime}$. Hence $g$ belongs to the kernel of $\chi$. But as $\chi$ is faithful on $G, g=e$. Therefore $(g, h)$ $=\left(e, e^{\prime}\right)$ and $\xi$ is faithful on $G \times_{\phi} H$.

Corollary (MAlCev) ${ }^{1}$ A connected solvable Lie group $G$ has a faithful representation if and only if $G=N A$, where $N$ is a closed, connected, simply connected invariant subgroup and $A$ is a connected, compact abelian subgroup such that $N \cap A=\{e\}$.

Suppose $G=N A$. For any $a \in A$ let $\phi(a)$ denote the automorphism of $N$ given by $\phi(a) n=a n a^{-1}(n \in N)$. Then it is easily seen that $(n, a) \rightarrow n a$ is an isomorphism of $N \times_{\phi} A$ onto $G$. Since $A$ is compact, it has a faithful representation. Hence by the above theorem it follows immediately that $G$ has a faithful representation.

In order to establish the converse we make use of the following lemma which follows easily from the results of Chevalley." 
Lemma 5.6 If $G$ is a connected solvable Lie group and $N$ a closed, connected invariant subgroup such that $G / N$ is compact, then there exists a compact connected abelian subgroup $A$ of $G$ such that $G=A N$ and $A \cap N$ is finite.

Returning to the corollary, suppose $G$ is linear. Since $g$ is solvable, we deduce in the usual way that every element $X \in[\mathfrak{g}, \mathfrak{g}]=\mathfrak{g}^{\prime}$ is nilpotent and therefore may be assumed to be subtriangular. Therefore by Lemmas 1 and 2 the group $G^{\prime}$ generated by $\mathfrak{g}^{\prime}$ is simply connected. Let $\dot{d}$ be the degree of $G$ and $G_{0}$ the group of all matrices in $G L(K, \dot{d})$ which have zero below the diagonal and 1 everywhere on the diagonal. By Lemma 2, $G^{\prime}$ is closed in $G_{0}$. However, since $G_{0}$ is clearly closed in $G L(K, \dot{d}), G^{\prime}$ is closed in $G L(K, \dot{d})$ and therefore in $G$. Let $x \rightarrow x^{*}$ denote the natural homomorphism of $G$ onto $G / G^{\prime}=G^{*}$. Since $G^{*}$ is abelian, $G^{*}=T^{*} V^{*}$ where $T^{*}$ and $V^{*}$ are connected subgroups, $T^{*}$ being compact and $V^{*}$ simply connected and $T^{*} \cap V^{*}$ $=\left\{e^{*}\right\}$. Let $N$ be the complete inverse image of $V^{*}$ in $G$. Since $N / G^{\prime}=V^{*}$ and $G^{\prime}$ are both simply connected, $N$ is simply connected and $G / N \cong T^{*}$ is compact. Therefore by Lemma $5, G=A N$ where $A$ is compact, connected, and abelian and $A \cap N$ is finite. Let $\sigma \in A \cap N$. Then $\sigma^{r}=e$ for some $r \geqq 1$. Then $\sigma^{*} \in V^{*}$ and $\left(\sigma^{*}\right)^{r}=e^{*}$. Since $V^{*}$ is simply connected and abelian, $\sigma^{*}=e^{*}$. Hence $\sigma \in A \cap G^{\prime}$. Since $X \rightarrow \exp X$ is a topological mapping of $\mathfrak{g}^{\prime}$ onto $G^{\prime}$, it follows that $\sigma \in G^{\prime}, \sigma^{r}=e$ implies $\sigma=e$. Hence $A \cap N=\{e\}$. The corollary is therefore proved.

Institute for Advanced Study

- This lemma was pointed out to me by Dr. G. D. Mostow. 